\title{
Tibiafraktur als operative Odyssee
}

\author{
Gert Muhr
}

30-jährige Patientin, Unfall August 2002

Diagnose kurzer distaler Unterschenkeldrehbruch links

Therapie Erstversorgung im regionalen Krankenhaus (Kondylenabstützplatte!)

Infektverlauf

Metallentfernung November 2002

11/2003 Vorstellung an einer Universitätsklinik wegen permanenter Schmerzen und langsamer Beinachsenverbiegung.

1/2004 Nach Diagnostik einer chronischen Osteitis dort stationäre Aufnahme, Resektion des erkrankten Tibiasegments und Fixateuranlage zum Segmenttransport

1/2005 Fixateurwechsel wegen Lockerung

5/2005 Fixateurabnahme und Oberschenkelgipsverband

6/2005 Spongiosaanlagerung und Plattenosteosynthese, sowohl an der Distraktionsstrecke als auch an der Dockingstelle

11/2005 Plattenbruch an der proximalen Tibia, deswegen Plattenwechsel und Anlagerung von Ossigraft

6/2006 Nach ausbleibender Heilung an der Dockingstelle Spongiosaplastiken

Abb. 2

Abb. 1

3/2007 Wegen Schraubenlockerung Reosteosynthese, Spongiosaplastik, Ossigraft und Vakuumversiegelung in einem weiteren Krankenhaus

8/2007 Bei der letzten Kontrolle dort Diagnose einer infizierten Pseudarthrose gestellt und eine Arthrodese des Fußes mittels Marknagelung vorgeschlagen

9/2007 Zu diesem Therapievorschlag Einholen einer weiteren Meinung an einer anderen Universitätsklinik. Dort wird neben der Marknagelarthrodese alternativ ein erneuter Fixateur mit Fibulaspan und BMP vorgeschlagen

9/2007 Eine dritte Meinung wird im selben Monat an einer Sportklinik eingeholt, wo zunächst eine Orthese, später eine schmale, kurze, ventrale Platte empfohlen wird

3/2008 Patientin stellt sich erstmals ohne weitere Operation im Vorzustand im Bergmannsheil vor, wo eine Osteosynthese empfohlen wird. Diese wird Ende März 2008 durchgeführt

9/2008 Vollbelastung möglich, die Pseudarthrose ist ausgeheilt

Abb. 6, 7

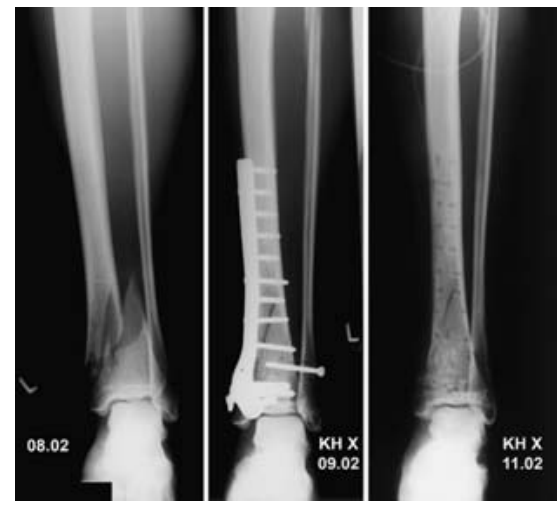

Abb. 1

Abb. 3

Abb. 4

Abb. 5

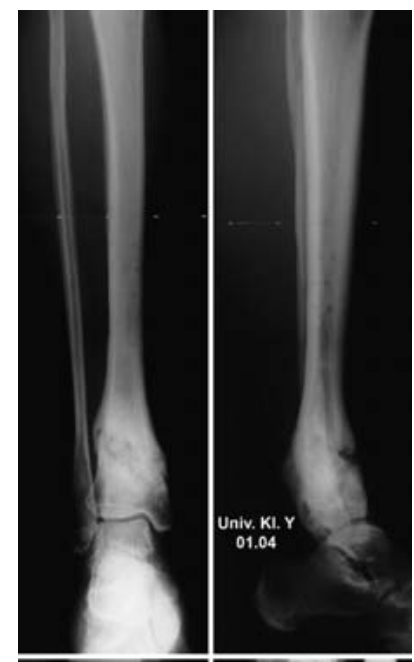

Abb. 8

Der Preis: 6 Jahre Krankheitsdauer, unzählige Operationen mit unterschiedlichen Implantaten, das Spongiosareservoir aufgebraucht, Beinverkürzung von $3 \mathrm{~cm}$.

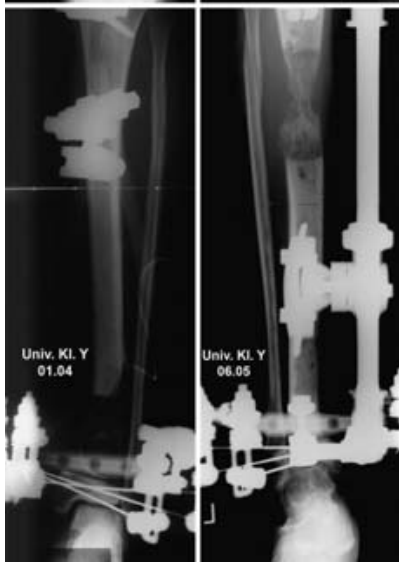

Abb. 2 


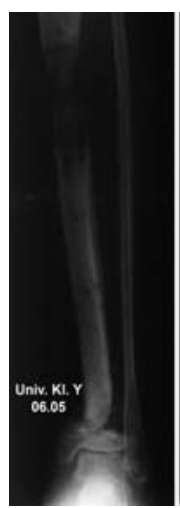

Abb. 3

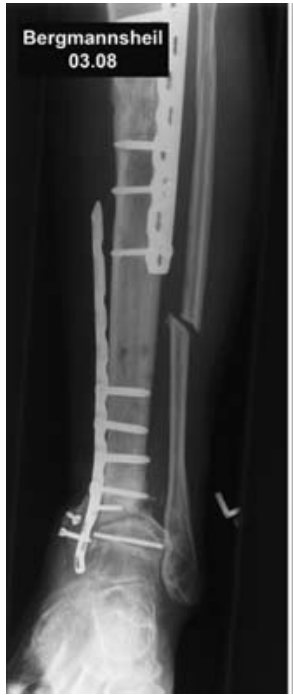

Abb. 6

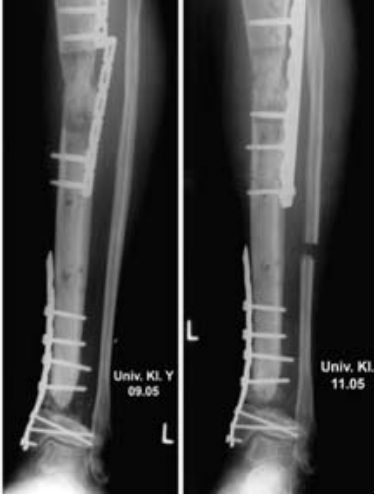

Abb. 4

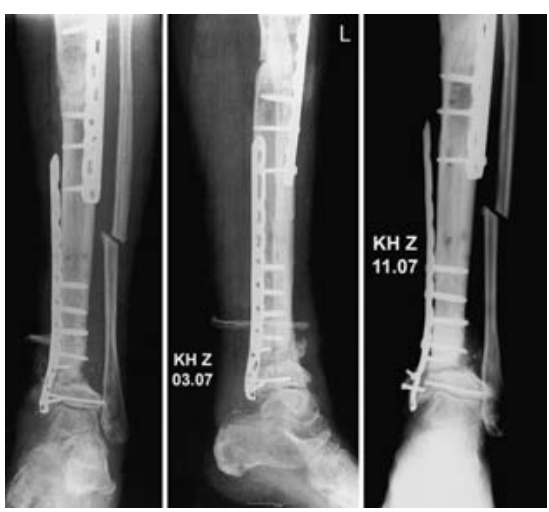

Abb. 5

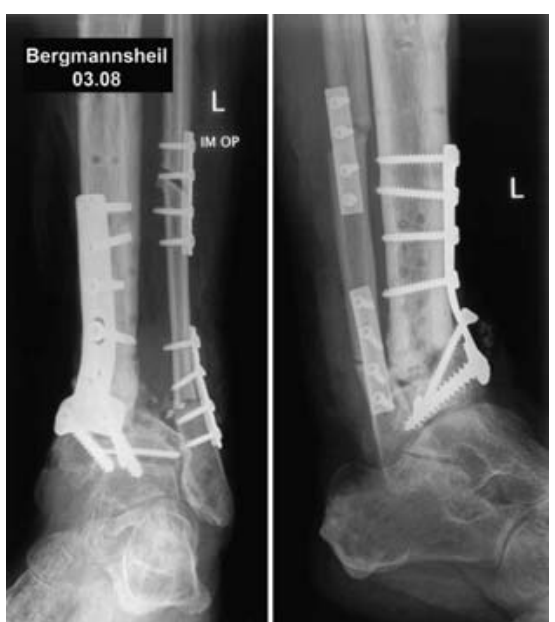

Abb. 7

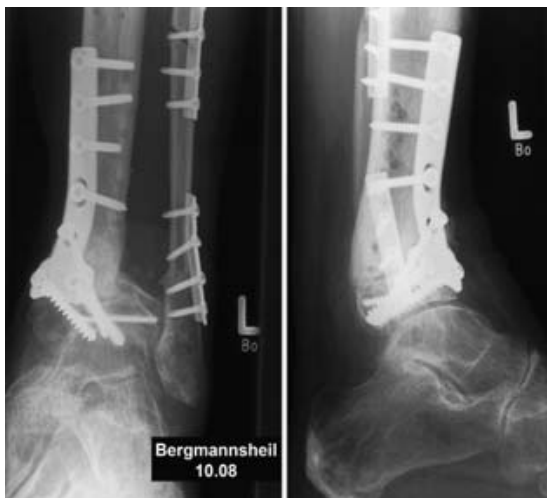

Abb. 8
Fazit

Korrektes Implantat bei einfacher Fraktur, raschere Entscheidungen, keine biomechanischen Kompromisse, klassische Standards beachten.
Prof. Dr. med. Gert Muhr

Ärztlicher Direktor

Chirurgische Klinik

Berufsgenossenschaftliches Universitätsklinikum Bergmannsheil Bürkle-de-la-Camp-Platz 1 44789 Bochum

E-Mail: chirurgie@bergmannsheil.de 